\title{
Papers
}

\section{Does dietary folate intake modify effect of alcohol consumption on breast cancer risk? Prospective cohort study}

\author{
Laura Baglietto, Dallas R English, Dorota M Gertig, John L Hopper, Graham G Giles
}

\begin{abstract}
Objective To evaluate the effect of dietary folate intake on the relation between alcohol consumption and breast cancer risk. Design Prospective cohort study.

Setting Melbourne, Australia.

Participants 17447 Anglo-Australian women resident in Melbourne, aged 40-69 years at recruitment in 1990-4, and followed up until 31 December 2003.

Main outcome measure Invasive breast cancers diagnosed during follow-up and ascertained through the Victorian cancer registry.

Results 537 invasive breast cancers were diagnosed. Compared with lifetime abstainers, the hazard ratio for breast cancer in women who consumed an average of $40 \mathrm{~g}$ or more of alcohol daily at baseline was 1.41 (95\% confidence interval 0.90 to 2.23). No direct association was found between dietary folate intake and risk of breast cancer, but a high folate intake mitigated the excess risk associated with alcohol. The estimated hazard ratio of an alcohol consumption of $40 \mathrm{~g}$ /day or more was 2.00 (1.14 to 3.49) for women with intakes of $200 \mu \mathrm{g} /$ day of folate and 0.77 (0.33 to 1.80) for $400 \mu \mathrm{g} /$ day of folate $(\mathrm{P}=0.04$ for interaction between alcohol and folate).

Conclusions An adequate dietary intake of folate might protect against the increased risk of breast cancer associated with alcohol consumption.
\end{abstract}

\section{Introduction}

Alcohol consumption is a known risk factor for breast cancer. Although the strength of the association is modest, its adverse effect on breast cancer is one of the most consistent findings among the many hypothesised dietary risk factors. ${ }^{1}$ Pooled analyses of both case-control studies and cohort studies confirm a linear dose-response relation between alcohol consumption and risk of breast cancer. ${ }^{23}$ However, the mechanisms involved in alcohol associated carcinogenesis are still unknown. Ethanol itself is not considered a carcinogen, but increasing evidence suggests that its metabolite acetaldehyde is responsible for the co-carcinogenic effects of alcohol consumption. ${ }^{4}$

Folate is a B vitamin necessary for the production of red blood cells and the synthesis and normal methylation of DNA. ${ }^{5}$ The role of folate in colorectal carcinogenesis has been widely studied, and an inverse dose dependent relation has been found ${ }^{6-8}$; studies also fairly consistently show that people with high alcohol intake and low folate intake are at higher risk of colorectal neoplasia compared with those with low alcohol and high folate intake. ${ }^{9}$ Some studies have also reported an inverse association between folate from diet or dietary supplements and risk of breast cancer ${ }^{10-13}$; the protective effect of folate on breast cancer is more pronounced for heavy drinkers. ${ }^{14-18}$ These findings suggest that folate and alcohol act in opposite directions in breast carcinogenesis and may interact with each other. To test this hypothesis, we used the Melbourne collaborative cohort study to investigate if the association between alcohol consumption and risk of breast cancer is modified by intake of dietary folate.

\section{Methods}

\section{Participants}

The Melbourne collaborative cohort study is a prospective cohort study of 41528 people (24 479 women) aged between 27 and 75 years at baseline (99.3\% aged 40-69). Recruitment took place between 1990 and 1994 in the Melbourne metropolitan area. Participants were recruited through the electoral rolls (registration to vote is compulsory for adults in Australia), advertisements, and community announcements in local media (such as television, radio, newspapers). Participants gave written consent and permission for the investigators to obtain access to their medical records.

We excluded women with a confirmed diagnosis of invasive breast cancer before baseline $(n=381)$ and women who reported a diagnosis of angina, heart attack, or diabetes at baseline $(n=1461)$, because their diets were not representative of the whole cohort and we could not rule out the possibility that they had changed their diet in response to a recent diagnosis. Other exclusions included women with missing data on alcohol intake or food items and extreme values for self reported total energy intake ( $<1$ st centile and $>99$ th centile) $(\mathrm{n}=439)$. Of the 22198 women left, $4751(21 \%)$ were born in Greece or Italy. These women had a lower incidence of breast cancer than other women; few of them consumed alcohol (61\% were lifetime abstainers compared with $31 \%$ of other women), and those that did had low levels of consumption (4\% consumed at least 20 $\mathrm{g}$ /day compared with $13 \%$ of other women). Thus, they would have added little information about the association between alcohol consumption and risk of breast cancer. To avoid confounding by country of birth, we restricted the analyses to the 17447 women born in Australia, New Zealand, or the United Kingdom.

\section{Assessment of alcohol consumption and diet}

At baseline we used a structured interview schedule to obtain information on potential risk factors, including age, sex, country of birth, education, reproductive history, and alcohol consumption. We asked participants a series of questions about their intake of alcoholic drinks. People who had never consumed at 
Table 1 Baseline characteristics of women in the Melbourne collaborative cohort study. Values are numbers (percentages) unless stated otherwise

\begin{tabular}{|c|c|c|c|c|c|}
\hline \multirow[b]{2}{*}{ Alcohol consumption } & \multicolumn{4}{|c|}{ Folic acid ( $\mu \mathrm{g} /$ day) } & \multirow[b]{2}{*}{ Total } \\
\hline & Mean (SD) & $<270^{\star}$ & $270-360 *$ & $>360^{*}$ & \\
\hline Abstainers† & 333 (131) & $1827 \quad(32)$ & $1733(30)$ & 1911 (32) & 5471 (31) \\
\hline Ex-drinkersł & 333 (133) & $189(3)$ & $165(3)$ & $198(3)$ & $552(3)$ \\
\hline $1-19 \mathrm{~g} / \mathrm{day}$ & $330(123)$ & $2918(51)$ & $3067(53)$ & $3121 \quad(53)$ & $9106(52)$ \\
\hline 20-39 g/day & $324(110)$ & $602(10)$ & $610(11)$ & $568(10)$ & $1780(10)$ \\
\hline$\geq 40 \mathrm{~g} / \mathrm{day}$ & 302 (105) & $222(4)$ & $182(3)$ & $134(2)$ & $538(3)$ \\
\hline
\end{tabular}

${ }^{*}$ Thirds of distribution of folate intake.

tWomen who never drank at least 12 alcoholic drinks in a year.

$\ddagger$ Women who drank at least 12 alcoholic drinks in a year, but did not drink at baseline.

least 12 alcoholic drinks in a year were considered lifetime abstainers. We asked non-lifetime abstainers at baseline about their current average quantity and frequency of intake of beer, wine, and spirits. We then asked them about the intake of alcoholic beverages on each day during the week before the interview (diary). Participants who were not lifetime abstainers but did not consume alcohol at baseline were classified as ex-drinkers. We used Australian food composition tables to calculate alcohol consumption from alcoholic beverages. ${ }^{19} \mathrm{We}$ categorised total alcohol consumption into lifetime abstainers, ex-drinkers, and current drinkers with low intake (1-19 g/day), medium intake (20-39 g/day), or high intake (40 g/day or more), according to guidelines of the Australian National Health and Medical Research Council. ${ }^{20}$

Participants completed a dietary questionnaire that included a 121 item food frequency questionnaire without portion sizes, specifically developed for the Melbourne collaborative cohort study. ${ }^{21}$ The questionnaire had 26 items on intake of vegetables, including fresh and cooked vegetables. We calculated nutrient intakes by using mean sex specific portion sizes from weighed food records. We used British data to calculate intake of folate. ${ }^{22}$ We calculated intake of energy from Australian food composition tables. ${ }^{19}$ Total energy intake included energy from the food frequency questionnaire and energy from alcohol. We calculated body mass index from measured height and weight.

\section{Cohort follow-up and ascertainment of invasive breast cancer cases}

Cases were women notified to the Victorian cancer registry with a first diagnosis of invasive breast cancer during follow-up to 31 December 2003. We did not count women with in situ breast cancer as cases.

We determined addresses and vital status of all participants by record linkage to electoral rolls, Victorian death records, and the national death index; from electronic phone books; and from responses to mailed questionnaires and newsletters. By the end of follow-up on 31 December 2003, 351 (2\%) of the women included in this analysis were known to have left Victoria and $627(4 \%)$ had died.

\section{Statistical analysis}

Follow-up began at baseline and continued until diagnosis of breast cancer, death, date of leaving Victoria, or 31 December 2003, whichever came first. We estimated hazard ratios by using Cox regression with age as the time metric. For both alcohol consumption and folate intake, we compared linear and more complex polynomial relations with the log hazard rate using fractional polynomials. ${ }^{23}$ Fractional polynomials are a method of analysing dose-response curves that make no a priori assumption about the shape of the curves. We also fitted a model in which alcohol intake was categorised according to the National Health and Medical Research Council categories. ${ }^{20}$ We studied the interaction between alcohol consumption and dietary folate intake. We compared non-nested models with the Akaike information criterion. ${ }^{24}$

We adjusted all analyses for total energy intake. Other potential confounders examined included education, body mass index, age at menarche, hormone replacement therapy, parity, and use of multivitamins. We did not include any of these variables in the final analyses, as their inclusion changed the estimated hazard ratios for the association with alcohol consumption by less than $5 \%$. We used S-PLUS 6.2 (Insightful Corporation, Seattle, WA) and Stata 8.2 (Stata Corporation, College Station, TX) for all statistical analyses.

\section{Results}

We identified 537 incident cases of invasive breast cancer (536 were histologically verified) over an average of 10.1 person years of follow-up between 1990 and 2003 in the 17447 women eligible for analysis. The mean age at baseline was 54.7 (SD 8.8) years; $36 \%$ of the women were under $50,16 \%$ between 50 and 55 , and $50 \%$ over 55 years.

Table 1 summarises the distribution of alcohol consumption and folate intake at baseline. Most women $(86 \%)$ drank on average less than $20 \mathrm{~g}$ of alcohol a day, and only 538 (3\%) reported an average daily intake of alcohol of at least $40 \mathrm{~g}$. The Spearman's correlation coefficient between the reported daily average alcohol consumption during the current decade of age at baseline and that from the diary of the week before baseline was 0.87 . The mean folate intake was 330 (SD 124) $\mu \mathrm{g} /$ day.

The comparison between the models of the log hazard rate using linear and fractional polynomials of current alcohol consumption provided no evidence for a departure from linearity $(\mathrm{P}=0.64$; null hypothesis: the association is linear). From the model in which alcohol consumption was fitted as a linear term, the hazard rate increased by 1.03 (95\% confidence interval 0.95 to 1.09) times for each additional $10 \mathrm{~g}$ a day intake of alcohol $(\mathrm{P}=0.36)$. Next, we modelled alcohol consumption as a categorical variable using the National Health and Medical Research Council categories $^{20}$ (table 2). The highest hazard ratio occurred in women who drank $40 \mathrm{~g}$ of alcohol a day or more; however, overall, the association of breast cancer incidence with alcohol was not significant $(\mathrm{P}=0.29)$.

The comparison between the regression models using linear and fractional polynomials of folate intake indicated that the more complex models did not fit better than the simple linear model ( $\mathrm{P}=0.27$; null hypothesis: the association is linear). However, folate was not significantly related to incidence of breast cancer (for $100 \mu \mathrm{g} /$ day increment of folate, relative risk $=1.01$ (0.93 to 1.10$) ; \mathrm{P}=0.79)$.

We evaluated whether folate modified the association between alcohol and breast cancer by fitting a model with an interaction between alcohol and folate. Our model of choice included alcohol as a categorical variable and folate as a continu- 
Table 2 Hazard ratios for association between average alcohol consumption at baseline and breast cancer in the Melbourne collaborative cohort study

\begin{tabular}{lccc} 
Alcohol consumption & Cases & Person years* & Hazard ratio† $\mathbf{( 9 5 \%}$ Cl) \\
\hline Abstainers $\ddagger$ & 171 & 56267 & Reference \\
\hline Ex-drinkers $\S$ & 16 & 5553 & $1.03(0.62$ to 1.73$)$ \\
\hline $1-19 \mathrm{~g} /$ day & 286 & 91274 & $1.12(0.93$ to 1.36$)$ \\
\hline $20-39 \mathrm{~g} /$ day & 43 & 17720 & $0.87(0.62$ to 1.22$)$ \\
\hline$\geq 40 \mathrm{~g} /$ day & 21 & 5313 & $1.41(0.90$ to 2.23$)$ \\
\hline
\end{tabular}

*Person years of follow-up of 17447 women.

†Adjusted for total energy and folate intake and fitted as linear variable in Cox's proportional hazard model with age as time metric.

‡Women who never drank at least 12 alcoholic drinks in a year

$\S$ Women who drank at least 12 alcoholic drinks in a year, but did not drink at baseline.

ous variable. We preferred this model to those including both variables as continuous because its results are easier to interpret. Table 3 shows estimates of the hazard ratios in relation to alcohol consumption for three values of folate intake. The values of folate were $200 \mu \mathrm{g} /$ day, $330 \mu \mathrm{g}$ /day (the mean value of folate intake), and $400 \mu \mathrm{g} /$ day, which is the Australian recommended daily intake for women in childbearing age..$^{25}$ Compared with abstainers who consumed $200 \mu \mathrm{g} /$ day of folate, the hazard ratio was twofold higher for those women who consumed the most alcohol (40 g/day or more), but the hazards ratios for lower levels of consumption were not elevated. None of the hazard ratios was elevated for women in any other categories of alcohol consumption or for women in the highest category of consumption who had higher levels of folate intake. The test for the interaction gave $\mathrm{P}$ values of 0.04 . The model in which alcohol and folate were both linear did not give a better fit $(\mathrm{AIC}=9138 v$ $\mathrm{AIC}=9138$ for the model with alcohol as categorical and folate as linear). The test for the interaction between alcohol and folate was not significant $(\mathrm{P}=0.42)$, probably because, as table 3 shows, the interaction between alcohol and folate was not a simple linear term.

\section{Discussion}

Overall in this prospective cohort study, we did not observe a significant association of breast cancer with either alcohol consumption or folate intake, but we found a significant interaction between alcohol and folate intakes. Women who had high alcohol consumption and low intake of folate had an increased risk of breast cancer, but those women who had high alcohol consumption and moderate to high levels of folate intake had no increased risk.

\section{Strengths and limitations}

The strengths of our study include its prospective nature, extensive information on potential confounding variables, minimal

Table 3 Hazard ratios* (95\% confidence intervals) for association between average alcohol consumption at baseline and breast cancer by intake of dietary folate in the Melbourne collaborative cohort study

\begin{tabular}{lccc} 
& \multicolumn{4}{c}{ Folic acid } \\
\cline { 2 - 4 } Alcohol consumption & $\mathbf{2 0 0} \boldsymbol{\mu \mathbf { g } / \text { day }}$ & $\mathbf{3 3 0} \boldsymbol{\mu} \mathbf{g} / \mathbf{d a y} \dagger$ & $\mathbf{4 0 0} \boldsymbol{\mu \mathbf { g }} / \mathbf{d a y}$ \\
\hline Abstainers $\ddagger$ & Reference & Reference & Reference \\
\hline Ex-drinkers $\S$ & $1.06(0.52$ to 2.16$)$ & $1.03(0.62$ to 1.73$)$ & $1.02(0.56$ to 1.87$)$ \\
\hline $1-19 \mathrm{~g} /$ day & $0.93(0.71$ to 1.23$)$ & $1.12(0.92$ to 1.35$)$ & $1.23(0.99$ to 1.53$)$ \\
\hline $20-39 \mathrm{~g} /$ day & $0.94(0.58$ to 1.54$)$ & $0.85(0.61$ to 1.20$)$ & $0.81(0.52$ to 1.25$)$ \\
\hline$\geq 40 \mathrm{~g} /$ day & $2.00(1.14$ to 3.49$)$ & $1.08(0.60$ to 1.93$)$ & $0.77(0.33$ to 1.80$)$ \\
\hline
\end{tabular}

*Hazard ratio from Cox's proportional hazard model with age as time metric. The model includes total energy, folate fitted as a linear variable, alcohol as categorical variable, and interaction between folate and alcohol consumption.

tMean of distribution of folate.

$\ddagger$ Women who never drank at least 12 alcoholic drinks in a year

$\S$ Women who drank at least 12 alcoholic drinks in a year, but did not drink at baseline. loss to follow-up, and virtually complete ascertainment of cases through the population cancer registry. Limitations include the small numbers of women with high levels of alcohol consumption and folate intake, use of a single measure of self reported alcohol consumption at baseline, and limitations in the assessment of folate intake. Random error in measuring alcohol consumption and folate intake at baseline may have led to conservative biases in the association between breast cancer incidence and alcohol and folate.

We measured folate intake at baseline, but changes in folate intake due to use of multivitamins, folate supplementation of foods, or change in dietary habits may have occurred during the follow-up period. Use of multivitamin supplements at baseline was not common and did not affect the hazard ratios, but we had no information on subsequent use of supplements containing folic acid. Since 1995, more than 100 foods have been approved for fortification with folate in Australia for the prevention of neural tube defects. ${ }^{25}{ }^{26}$ Changes in serum folate concentration following the voluntary fortification of food in Australia have been shown to be very small, especially compared with those in the United States, where folate fortification has been mandatory. ${ }^{27}$

\section{Comparison with other studies}

Epidemiological evidence for an effect of alcohol consumption on risk of breast cancer has been summarised in a collaborative reanalysis of individual data from 53 epidemiological studies. ${ }^{3}$ Compared with women who reported drinking no alcohol, the relative risk of breast cancer was 1.46 (95\% confidence interval 1.33 to 1.61) for those consuming on average $\geq 45 \mathrm{~g} /$ day, a result in general agreement with our finding of a hazard ratio of 1.41 (0.90 to 2.23) for those consuming $\geq 40 \mathrm{~g} /$ day. According to the collaborative reanalysis, the relative risk of breast cancer increased by $7 \%$ for each additional $10 \mathrm{~g} /$ day intake of alcohol. Our weaker association of a $3 \%$ increase is consistent with another reanalyses of six prospective studies restricted to women who drank less than $60 \mathrm{~g} /$ day of alcohol, which found increases of $3-16 \%$ across individual studies. ${ }^{2}$

Few other studies have investigated the interaction between folate and alcohol. ${ }^{14-17}$ Two reports showed a higher risk of breast cancer in women with high intake of alcohol and low intake of folate, ${ }^{14}{ }^{17}$ whereas the other two studies focused on the protective effect of folate when associated with high consumption of alcohol. ${ }^{15} 16$

\section{Possible mechanisms}

Consistent evidence from animal experiments shows that ethanol modulates chemically induced carcinogenesis. ${ }^{4}$ The mechanisms by which alcohol induces carcinogenesis are hypothesised to include the induction of cytochrome P-4502E1 (CYP2E1), which metabolises ethanol to acetaldehyde and is also involved in the metabolism of various procarcinogens; nutritional deficiencies, including folate deficiency caused by either low intake or destruction by acetaldehyde; interactions with retinoids with effects on cellular growth and differentiation; changes in DNA methylation; alteration in the immune system; and alcohol mediated increases in oestradiol. ${ }^{4}$ Acetaldehyde is carcinogenic and mutagenic, binds to DNA and proteins, destroys folate, and causes hyperproliferation. ${ }^{28}$ How these mechanisms are involved in breast carcinogenesis is not clear, but the impact of alcohol on hormonal status and cumulative exposure to oestrogens, particularly oestradiol, is likely to be a major contributor to the risk of breast cancer. ${ }^{1}$ It is also not clear whether the modifying effect of folate is an early or late stage effect or whether total dose is important. ${ }^{7}$ 


\section{What is already known on this topic}

Alcohol consumption is a known risk factor for breast cancer

Some studies have reported that folate is inversely associated with breast cancer risk

Whether folate intake can mitigate the adverse effects of alcohol on risk of breast cancer is not well established

\section{What this study adds}

This study strengthens the evidence that an adequate dietary intake of folate might protect against the increased risk of breast cancer associated with alcohol consumption

\section{Conclusion}

Our results support the hypothesis that alcohol consumption may increase the risk of breast cancer through an interaction with folate and suggest that any adverse effect of alcohol consumption may be reduced by sufficient dietary intake of folate.

This study was made possible by the contribution of many people, including the original investigators and the diligent team who recruited the participants and who continue working on follow-up. Finally, we thank the many thousands of Melbourne residents who continue to participate in the study.

Contributors: GGG, DMG, and JLH were responsible for the initial study design. DRE and GGG supervised the conduct of the study. LB did the data analysis. All of the authors contributed to the final report. All the authors are guarantors.

Funding: Cohort recruitment was funded by VicHealth and the Cancer Council Victoria. This analysis was partly funded by grants from the National Health and Medical Research Council (grants No 209057 and 251533), the National Breast Cancer Foundation (grant No: KCF20), and the Australian Brewers Foundation.

Competing interests: None declared.

Ethical approval: The Cancer Council Victoria's human research ethics committee approved the study protocol.

1 Singletary KW, Gapstur SM. Alcohol and breast cancer: review of epidemiologic and experimental evidence and potential mechanisms. JAMA 2001;286:2143-51.

2 Smith-Warner SA, Spiegelman D, Yaun SS, van den Brandt PA, Folsom AR, Goldbohm RA, et al. Alcohol and breast cancer in women: a pooled analysis of cohort studies. JAMA 1998;279:535-40.

3 Hamajima N, Hirose K, Tajima K, Rohan T, Calle EE, Heath CW Jr, et al. Alcohol, tobacco and breast cancer-collaborative reanalysis of individual data from 53 epidemiological studies, including 58,515 women with breast cancer and 95,067 women without the disease. Br J Cancer 2002;87:1234-45.

4 Poschl G, Seitz HK. Alcohol and cancer. Alcohol Alcohol 2004:39:155-65.

5 Fairfield KM, Fletcher RH. Vitamins for chronic disease prevention in adults: scientific review. JAMA 2002;287:3116-26.

6 Kim Y-I. Folate and carcinogenesis: evidence, mechanisms, and implications. J Nutr Biochem 1999:10:66-88.
7 Kim YI. Folate and cancer prevention: a new medical application of folate beyond hyperhomocysteinemia and neural tube defects. Nutr Rev 1999;57:314-21.

8 Mason JB, Levesque T. Folate: effects on carcinogenesis and the potential for cancer chemoprevention. Oncology 1996;10:1727-36, 1742-3; discussion 1743-4.

9 Giovannucci E. Alcohol, one-carbon metabolism, and colorectal cancer: recent insight from molecular studies. J Nutr 2004;134:2475-81S.

10 Shrubsole MJ, Jin F, Dai Q, Shu XO, Potter JD, Hebert JR, et al. Dietary folate intake and breast cancer risk: results from the Shanghai breast cancer study. Cancer Res 2001;61:7136-41.

11 Ronco A, De Stefani E, Boffetta P, Deneo-Pellegrini H, Mendilaharsu M, Leborgne F. Vegetables, fruits, and related nutrients and risk of breast cancer: a case-control study in Uruguay. Nutr Cancer 1999;35:111-9.

12 Freudenheim JL, Marshall JR, Vena JE, Laughlin R, Brasure JR, Swanson MK, et al. Premenopausal breast cancer risk and intake of vegetables, fruits, and related nutrients. J Natl Cancer Inst 1996;88:340-8.

13 Graham S, Hellmann R, Marshall J, Freudenheim J, Vena J, Swanson M, et al. Nutritional epidemiology of postmenopausal breast cancer in western New York. Am J Epidemiol 1991;134:552-66.

14 Zhang S, Hunter DJ, Hankinson SE, Giovannucci EL, Rosner BA, Colditz GA, et al. A

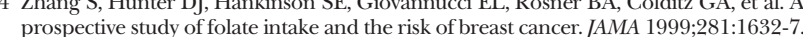

15 Negri E, La Vecchia C, Franceschi S. Re: dietary folate consumption and breast cancer Negri E, La Vecchia C, Franceschi S. Re:
risk.J Natl Cancer Inst 2000;92:1270-1.

16 Rohan TE, Jain MG, Howe GR, Miller AB. Dietary folate consumption and breast cancer risk. J Natl Cancer Inst 2000;92:266-9.

17 Sellers TA, Kushi LH, Cerhan JR, Vierkant RA, Gapstur SM, Vachon CM, et al. Dietary folate intake, alcohol, and risk of breast cancer in a prospective study of postmenopausal women. Epidemiology 2001;12:420-8.

18 Sellers TA, Grabrick DM, Vierkant RA, Harnack L, Olson JE, Vachon CM, et al. Does folate intake decrease risk of postmenopausal breast cancer among women with a family history? Cancer Causes Control 2004;15:113-20.

19 Lewis J, Milligan G, Hunt A. NUTTAB95 nutrient data table for use in Australia. Canberra: Australian Government Publishing Service, 1995

20 Pols RG, Hawks DV. Is there a safe level of daily consumption of alcohol for men and women? Recommendations regarding responsible drinking behaviour. Canberra: National Health and Medical Research Council, 1992.

21 Ireland P, Jolley D, Giles GG, O'Dea K, Powles J, Rutishauser I, et al. Development of the Melbourne FFQ: a food frequency questionnaire for use in an Australian prospective study involving an ethnically diverse cohort. Asia Pacific J Clin Nutr 1994:3:19-31.

22 Holland B, Welch AA, Unwin ID, Buss DH, Paul AA, Southgate DAT. McCance and Widdowson's the composition of foods. 5th ed. Cambridge: Royal Society of Chemistry, 1993.

23 Altman DG, Royston P. Regression using fractional polynomials of continuous covariates: parsimonious parametric modelling. Appl Stat 1994;43:429-67.

24 Akaike H. Information theory and an extension of the maximum likelihood principle. 2nd International Symposium on Information Theory 1973:267-81.

25 Abraham B, Webb K. Interim evaluation of the voluntary folate fortification policy. Australian Food and Nutrition Monitoring Unit, 2000. ftp://www.sph.uq.edu.au/pdf.ftp/ P08b.pdf (accessed 5 Aug 2005).

26 Report of the Expert Panel on Folate Fortification. Endorsed at the 117th session of the NHMRC, Sydney, 1-2 June 1994. Canberra: Australian Government Publishing Service, 1995 .

27 Metz J, Sikaris KA, Maxwell EL, Levin MD. Changes in serum folate concentrations following voluntary food fortification in Australia. Med J Aust 2002;176:90-1.

28 Seitz HK, Matsuzaki S, Yokoyama A, Homann N, Vakevainen S, Wang XD. Alcohol and cancer. Alcohol Clin Exp Res 2001;25:137-43.

$$
\text { (Accepted 27 June 2005) }
$$

doi $10.1136 /$ bmj.38551.446470.06

Cancer Epidemiology Centre, The Cancer Council Victoria, Melbourne, 100 Drummond Street, Carlton, Victoria 3053, Australia

Drummond Street, Carlton, Victoria
Laura Baglietto senior research fellow

Dallas R English associate director

Graham G Giles director

Centre for Genetic Epidemiology, University of Melbourne, Melbourne Dorota M Gertig senior research fellow

John L Hopper director

Correspondence to: L Baglietto laura.baglietto@cancervic.org.au 